Short Communication

\title{
Sick Day Management in Type 1 Diabetes in Children
}

\author{
Kashyap $\mathbf{L}^{1}$ \\ ${ }^{1}$ Dr Liladhar Kashyap, MBBS, MD, Fellow Endocrinology Children's hospital Louisiana state university Health Sciences center \\ 200 Henry clay ave, New Orleans, LA-70118, USA.
}

Address for Correspondence: Dr. D Liladhar Kashyap, Email: 1kashy@1suhsc.edu

\begin{abstract}
New onset of diabetes in a child results a significant psychosocial stress in a family. However, when any illness occurs in someone with diabetes, the potential for either hyperglycemia, hyperglycemia with ketoacidosis or hypoglycemia exists and requires education and treatment to prevent exacerbation or even possible death
\end{abstract}

Key words: diabetes, Sick Day Management, Diabetes in Children

\section{Case study}

Suraj is 12 yrs of age and has been recently diagnosed with Type 1 diabetes. He has commenced multiple daily insulin regimen of short acting insulin analogue before each meal and long acting insulin Levemir once daily at bed time. Three months following diagnosis Suraj's mother calls you to say that he has been vomiting and not eating since last night and she has stopped her insulin because he is not able to keep any food down. Her blood glucose is $300 \mathrm{mg} / \mathrm{dl}$. He usually takes 5-8 units of short acting insulin before meals and 18 units of Levemir at bed time with total Insulin of 35 units/day. What would be your advice?

New onset of diabetes in a child results a significant psychosocial stress in a family. Managing diabetes at home has been a challenge for many parents because of limited supervision by parents due to their busy schedule, less social support by family and friends, frequent omissions of insulin by patient. This problem is further compounded by a common notion of person who thinks that their child would need not any insulin if he is not eating. Children whose diabetes is under good metabolic control should not experience more illness or infections than children without diabetes. However, when any illness occurs in someone with diabetes, the potential for either hyperglycemia, hyperglycemia with ketoacidosis or hypoglycemia exists and requires education and treatment to prevent exacerbation or even possible death.

Many illnesses, especially those associated with fever, raise blood glucose levels because of higher levels of stress hormones promoting gluconeogenesis and insulin resistance.

International Journal of Medical Research and Review
Sometimes some illnesses associated with vomiting and diarrhea may lower BG with the possibility of hypoglycemia rather than hyperglycemia. Decreased food intake, poor absorption and slower emptying of stomach during gastroenteritis may contribute to hypoglycemia. Sometimes there are increased insulin requirements during the incubation period of an infection for a few days before the onset of the illness.

Education about the effects of intercurrent illness ("sick days") is a critical component of diabetes management at home and must be adapted to the educational abilities and the treatment possibilities of the particular situation in different parts of the world. Re-education is also necessary since forgetting the "rules" (i.e. when to check urine or blood ketone levels, for instance), or forgetting to maintain such emergency supplies are also common.

\section{General principles}

1. During sick days, do not stop insulin.

2. During sick days, the insulin dose may need to be increased or decreased temporarily.

3. Blood sugar and urine ketones should be monitored every 2-3 hrs. Blood ketene testing is superior to urine ketone testing adult studies have shown that the time delay to diagnosis of ketosis is significantly longer for ketonuria than for plasma ketonemia and that a urinary ketone test can remain positive more than 24 hs after resolution of an episode of ketoacidosis.

4. Fluid and Electrolytes

Available online at: $\underline{w w w . i j m r r . i n}$ 31 | P a g e 
Short Communication

* If the blood sugar is below $120 \mathrm{mg} / \mathrm{dl}$

FLUIDS: need to contain extra glucose such as soda or simple sugar and electrolyte (sodium chloride) containing fluids (like the WHO ORS solution) should be added.

The child should continue with glucose-containing drinks until the blood sugar is above $120 \mathrm{mg} / \mathrm{dl}$

\section{* If the blood glucose is between 120 and $180 \mathrm{mg} / \mathrm{dl}$}

FLUIDS: need to contain approximately $15 \mathrm{~g}$ of carbohydrates such as half cup of ginger ale or cola, half cup of juice or half Popsicle.

FOODS: need to have at least $15 \mathrm{~g}$ of carbohydrates per serving. If the child can not eat normal meals, possible sick day foods include: half cup of ice cream or sherbet, once slice of toast.

\section{* If the blood glucose is above $180 \mathrm{mg} / \mathrm{dl}$}

FLUIDS: need to have no calories such as diet soda, unsweetened tea, or water.

FOODS: need to be small servings as tolerated.

5. Additional insulin is usually provided based upon one of several formulas:

a. $5-10 \%$ of total daily dose of insulin (or $0.05-0.1 \mathrm{U} / \mathrm{kg}$ ) as short or rapid-acting insulin repeated every 2-4 hours based upon elevated blood glucose results if there are negative or small amounts of ketones.

b. $10-20 \%$ of total daily dose of insulin (or $0.1 \mathrm{U} / \mathrm{kg}$ ) as short or rapid-acting insulin repeated every 2-4 hours based upon elevated blood glucose results if there are moderate or large amounts of ketones.

c. The basal insulin, especially if insulin pump treatment is being used, may also need to be increased depending upon individual illness requirements, blood glucose and ketone monitoring results.

6. The diabetes care team should provide clear guidance to patients and families on how to manage diabetes during inter current illnesses to avoid the complications of ketoacidosis, dehydration, uncontrolled or symptomatic hyperglycaemia

International Journal of Medical Research and Review and hypoglycaemia. Periodic review and re-education should include instruction on recognition and treatment during sick days.

7. When vomiting occurs in a child with diabetes, it should always be considered a sign of insulin deficiency until proven otherwise.

8. More frequent monitoring of blood or urine glucose as well as blood or urine ketones is required during sick days.

9. If home glucose and/or ketone monitoring is unavailable, then urgent or emergent consultation with the health care team should be arranged while attempts at maintaining hydration are utilised.

10. Sources of simple sugar and electrolyte (sodium chloride) containing fluids (like the WHO ORS solution) must be available for emergency use during sick days. This should include clean/boiled/purified cool water to provide hydration. Sugar containing liquids are helpful to prevent hypoglycemia and starvation ketosis.

11. Any underlying illness causing metabolic derangement should be diagnosed and treated.

12. Appropriate treatment of fever should be instituted to decrease risk of dehydration.

13. Appropriate treatment of nausea and vomiting would include correction of the primary illness, identification and treatment of hypoglycaemia and identification and treatment of insulin deficiency or insulin resistance associated with the primary illness. Pump failure can be a cause of ketosis and nausea/vomiting.

14. Recognition of ongoing or more severe dehydration and potential for decompensated diabetic ketoacidosis and coma must be taught, recognised by the patient and family caregivers and means of contacting health care professionals for institution of intravenous or other parental rehydration established. Actual weight with a scale several times each day will help to identify more serious dehydration and fluid losses requiring non-home care and parenteral rehydration.

15. Written guidelines for sick day management should be available and individualized for each child and adolescent with appropriate identification of who in the family will provide support and assistance under such circumstances. 
Short Communication

Education and periodic re-education of sick day management should occur at least annually.

16. Sometimes illness is associated with hypoglycaemia rather than hyperglycaemia, especially if there is a gastrointestinal illness rather than a respiratory illness.

Blood glucose monitoring is important for recognition of when this occurs. With gastroenteritis, insulin doses usually need to be decreased, but there is a risk of subsequent ketoacidosis if they are decreased too much. Sugarcontaining drinks should be given in small sips, along with small doses of insulin.

If ketones develop, this is an indication that the child needs more carbohydrates (and more insulin). Treatment of hypoglycaemia includes rapid-acting glucose, sucrose and/or fructose tablets, liquids or intravenous glucose.

17. Mini-glucagon dose regimens can also be used.

\section{References}

1. Brink S, Laffel L, Likitmaskul S, Liu L, Maguire AM, Olsen B, et al. Sick day management in children and adolescents with diabetes. Pediatr Diabetes2009 Sep;10 Suppl 12:146-53.

2. LifshitzF. Pediatric Endocrinology: Informa Healthcare; 2007

\section{How to cite this article?}

Kashyap L. Sick Day Management in Type 1 Diabetes in Children. Int J Med Res Rev 2013;1(1):31-33. doi: 10.17511/ijmrr.2013.i01.06. 